\title{
Drug-eluting Stents: Durchbruch oder Zeitbombe?
}

Lüscher, Thomas F ; Silber, Sigmund

DOI: https://doi.org/10.1007/s00059-007-3018-8

Other titles: Drug-eluting stents: breakthrough or time bomb

Posted at the Zurich Open Repository and Archive, University of Zurich ZORA URL: https://doi.org/10.5167/uzh-156411

Journal Article

Published Version

Originally published at:

Lüscher, Thomas F; Silber, Sigmund (2007). Drug-eluting Stents: Durchbruch oder Zeitbombe? Herz, 32(4):265-267.

DOI: https://doi.org/10.1007/s00059-007-3018-8 


\title{
Drug-eluting Stents: Durchbruch oder Zeitbombe?
}

\author{
Thomas F. Lüscher' ${ }^{1}$, Sigmund Silber ${ }^{2}$
}

Die koronare Herzkrankheit (KHK) und der akute Myokardinfarkt (AMI) gehen mit einer hohen Morbidität und Mortalität einher [1]; die perkutane Koronarintervention (PCI) stellt die bevorzugte Behandlungsmethode bei Patienten mit stabiler, symptomatischer KHK und AMI dar [2]. Die PCI wurde initial allein in Form einer Ballonangioplastie zur Dehnung eines verengten Gefäßsegments verwendet. Allerdings stellte die Entwicklung von Restenosen ein Hauptproblem für den Langzeiterfolg der Ballonangioplastie dar, eine Komplikation, welche mit einer Häufigkeit von ca. 20-50\% nach Intervention auftrat [3]. Mit der Einführung von Stents konnte die Restenoserate im Vergleich zur Ballonangioplasie deutlich gesenkt werden $[4,5]$; da allerdings immer noch $15-20 \%$ der Patienten eine Restenose entwickelten, wurden die „nackten“ Metallstents (,bare-metal stents“ [BMS]) mit biologisch aktiven Substanzen beschichtet, welche nach Implantation in die Gefäßwand diffundieren (sog. Drug-eluting Stents [DES]). Sinn und Zweck dieser Substanzen war es, die Migration und Proliferation glatter Gefäßmuskelzellen, welche entscheidend zur Ausbildung der Restenose beitragen, zu hemmen. Erwartungsgemäß konnte durch den Einsatz von DES die Häufigkeit der Restenose und von Reinterventionen auf $<10 \%$ reduziert werden [6] - also ein Durchbruch für die interventionelle Kardiologie. Ramcharitar et al. befassen sich in ihrer Arbeit eingehend mit dem angiographischen und klinischen Erfolg dieser neuen Behandlungsform in der interventionellen Kardiologie und besprechen zudem Behandlungen der selteneren In-Stent-Restenose nach Implantationen solcher Stents [7].

Bereits nach Einführung der BMS wurden sich die interventionellen Kardiologen des Problems der Stentthrombose bewusst; vor allem wegen der hohen Mortalität gilt sie als eine der meistgefürchteten Komplikationen in der interventionellen Kardiologie. Mit der Einführung neuer Thrombozytenaggregationshemmer wie Ticlopidin oder Clopidogrel in Kombination mit Aspirin konnte die Häufigkeit der Stentthrombose bei stabilen Patienten auf $<1 \%$ gesenkt werden [8]. Meist kam es innerhalb der ersten 10 Tage zu dieser Komplikation [9], während Stentthrombosen nach Implantationen von BMS nach dem 1. Monat selten auftreten [10]. In der Tat waren späte Stentthrombosen in einer großen, mehr als 6000 Patienten umfassenden Studie bei lediglich $0,2 \%$ der Patienten zu beobachten [11]. Bald zeigte sich auch, dass die DES zwar die Restenoserate deutlich verminderten, im Vergleich zu den BMS die Stentthromboserate aber nicht reduzierten [11-15]. Einige „real world“-Register legten sogar nahe, dass die Thromboserate nach DES-Implantationen gegenüber BMS leicht erhöht sein könnte [16, 17]. In ihrem Beitrag gehen Kaiser und Pfisterer umfassend auf die Frage ein, ob Stentthrombosen bei Patienten mit DES gehäuft sind [18]. Der Beitrag von Steffel und Tanner beleuchtet eingehend biologische Effekte von DES in der Koronarzirkulation, welche für diese Komplikation von Bedeutung sein könnten [19]. Die genannte Arbeit wird durch jene von Takano und Mizuno erweitert, welche über angioskopische Befunde nach der Implantation von DES berichten [20]. Diese Untersuchungen zeigen, dass - wie von der Grundlagenforschung erwartet - die Reendothelialisierung sich nach Implantation von DES schlechter entwickelt als nach BMS. Tatsächlich kommt es bei vielen Patienten zu einer unvollständigen Abdeckung der Stentstreben durch Endothelzellen. Diese Befunde werden weiter durch die Arbeiten von Virmanis Gruppe unterstützt. Nakazawa et al. zeigen in ihrer Übersichtsarbeit über die pathologischen Befunde nach Implantation von DES klar, dass erhebliche Teile der implantierten Stents nicht durch Gewebe abgedeckt werden und die Reendothelialisierung ebenso gehemmt wird wie die Proliferation von Gefäßmuskelzellen [21]. Die ungedeckten Stellen implantierter Stents könnten Ausgangspunkt später Stentthrombosen sein, wie sie heute zwar immer noch selten, aber doch häufiger als früher in vielen Herzkatheterlabors beobachtet werden. Aufgrund dieser Befunde ist natürlich insbesondere die Nachbehandlung bei DES von großer Bedeutung. Eberli und Roffi beleuchten die Möglichkeiten medikamentöser Therapie bei dieser Patientengruppe und weisen vor allem auf die Notwendigkeit einer mindestens 1-jährigen doppelten plättchenhemmenden Therapie hin [22], wofür es allerdings keine harten Daten im Sinne von randomisierten Studien gibt. Ebenfalls werden Nutzen und Risiko einer solchen Therapie, besonders wenn sie sich über einen längeren Zeitraum erstreckt, besprochen. Außerdem ge-
${ }^{1}$ Klinik für Kardiologie, HerzKreislaufZentrum, UniversitätsSpital Zürich, Schweiz, ${ }^{2}$ Kardiologische Praxis und Praxisklinik, München. Akademische Lehrpraxis der Ludwig-MaximiliansUniversität München.
Herz 2007;32:265-7 DOI 10.1007/ s00059-007-3018-8 
hen die Autoren auf das Management von Patienten nach DES-Implantation ein, bei welchen nichtkardiale chirurgische Eingriffe geplant werden. Diese Fragen sind vor allem deshalb interessant und von Bedeutung, als einige Untersuchungen darauf hinweisen, dass das Absetzen von Clopidogrel bei der Entstehung der Stentthrombose eine gewisse Rolle spielt.

Aufgrund der Befunde der letzten Monate stellt sich die Frage, welche Neuentwicklungen in dieser Situation von besonderem Interesse wären. Sicher wäre es wünschenswert, Substanzen zu entwickeln, welche spezifisch die Gefäßmuskelzellen in ihrem Wachstum hemmen, ohne Migration und Homing lokaler und zirkulierender Endothelzellen und Endothelvorstufenzellen zu beeinträchtigen. Damit könnte eine ideale Abheilung der Gefäßwand nach DES-Implantation erreicht werden. Leider sind solche idealen Substanzen im Moment noch nicht verfügbar, obschon Steffel und Tanner in ihrem bereits erwähnten Beitrag über biologische Effekte von DES [19] auf mögliche Entwicklungen eingehen. Zudem wäre es denkbar, mit spezifischen Antikörpern das Homing von endothelialen Progenitorzellen und damit die Reendothelialisierung nach DES-Implantation $\mathrm{zu}$ verbessern. Erste Untersuchungen entsprechend gestalteter Stents laufen derzeit. Erste Ergebnisse über die Wirksamkeit und Sicherheit solcher biologisch aktiver Stents aus nichtrandomisierten Studien liegen vor [23].

Schließlich wäre es denkbar, durch die Absorption der Stentstreben selbst die Abheilung der durch die Implantation gesetzten Wunde in der Gefäßwand zu verbessern. Erbel et al. gehen umfassend auf die klinischen Möglichkeiten absorbierbarer Stents ein, insbesondere auf den absorbierbaren Magnesiumstent [24].

Insgesamt zeigen die Beiträge dieses Schwerpunkthefts klar, dass die DES ein bedeutender Durchbruch für die interventionelle Kardiologie sind, der die Therapiemöglichkeiten auch komplexer Läsionen und die Nachhaltigkeit der Revaskularisation deutlich verbessert hat. Die Stentthrombose allerdings ist ein Problem, das verstanden und in $\mathrm{Zu}$ kunft durch neuere, noch bessere Stents verhindert werden sollte. Wenn auch die Frage der Häufigkeit von Stentthrombosen nach DES-Implantationen immer noch nicht klar ist, weisen doch einige Daten, vor allem Register, aus dem Alltag interventioneller Kardiologen darauf hin, dass diese Komplikation etwas häufiger auftreten dürfte als bei den ,nackten“ BMS. Sicher ist, dass DES durch ihre biologischen Eigenschaften bzw. diejenigen der eluierten Substanzen die Stentthrombogenität erhöhen, zum einen durch die Induktion des Tissue-Faktors, zum anderen durch die Beeinträchtigung der Reendothelialisierung. Die gegenwärtigen klinischen Daten erlauben allerdings keine endgültige Interpretation, ob das Thromboserisiko unter einer doppelten Plättchenhemmung bei DES im Vergleich zu BMS wirklich erhöht ist. Jedoch ist von Bedeutung, dass eine vorzeitige Beendigung einer solchen Therapie mit einem deutlich erhöhten Risiko für spätere Stentthrombosen nach DES vergesellschaftet ist. Damit stellt sich die Frage, ob eine längerfristige, ggf. mehrjährige duale Plättchenhemmung sinnvoll wäre. Dabei ist allerdings zu berücksichtigen, dass das erhöhte Blutungsrisiko gegenüber dem Nutzen einer verminderten Stentthromboserate abzuwägen ist. In der PCI-CURE-Studie führte die doppelte Plättchenhemmung zu einem Anstieg der schweren invalidisierenden oder lebensbedrohlichen Blutungen um 0,2\% und der übrigen Blutungen um 1,4\% über 1 Jahr [25]. In der CHARISMA-Studie erhöhte die doppelte Plättchenhemmung über einen Zeitraum von 18 Monaten die tödlichen Blutungen um $0,1 \%$ und die schweren Blutungen um 0,4\% [26]. $\mathrm{Ob}$ bei einer erwarteten Stentthromboserate von ungefähr 0,6\% pro Jahr [27] eine doppelte Plättchenaggregationshemmung bei allen Patienten über mehrere Jahre von Nutzen ist, müsste daher zuerst in einer großen Studie geprüft werden. Immerhin ist eine solche Therapie bei denjenigen Patienten in Betracht $\mathrm{zu}$ ziehen, die bereits eine Stentthrombose durchgemacht haben und die eine bekannte erhöhte Thrombogenität aufweisen. Bei diesen Patienten sollten insbesondere andere blutungsfördernde Therapien, wie die nichtsteroidalen Antiphlogistika, vermieden und ggf. ein Protonenpumpenblocker eingesetzt werden [28].

Wenn auch noch viele Fragen offen bleiben, hoffen wir, mit diesem Schwerpunktheft der geneigten Leserin und dem geneigten Leser für den Praxisalltag wichtige Informationen vermitteln zu können, sei es für die interventionelle Behandlung oder Nachbetreuung von Patienten mit KHK oder jenen nach AMI.

\section{Literatur}

1. Chambless L, Keil U, Dobson A, et al. Population versus clinical view of case fatality from acute coronary heart disease: results from the WHO MONICA Project 1985-1990. Multinational MONItoring of trends and determinants in CArdiovascular disease. Circulation 1997;96:3849-59.

2. Van de Werf F, Ardissino D, Betriu A, et al. Management of acute myocardial infarction in patients presenting with ST-segment elevation. The Task Force on the Management of Acute Myocardial Infarction of the European Society of Cardiology. Eur Heart J 2003;24:28-66.

3. Horlitz M, Sigwart U, Niebauer J. Fighting restenosis after coronary angioplasty: contemporary and future treatment options. Int J Cardiol 2002;83:199-205.

4. Serruys PW, de Jaegere P, Kiemeneij F, et al. A comparison of balloon-expandable-stent implantation with balloon angioplasty in patients with coronary artery disease. Benestent Study Group. N Engl J Med 1994;331:489-95 
5. Fischman DL, Leon MB, Baim DS, et al. A randomized comparison of coronary-stent placement and balloon angioplasty in the treatment of coronary artery disease. Stent Restenosis Study Investigators. N Engl J Med 1994;331: 496-501.

6. Serruys PW, Kutryk MJ, Ong AT. Coronary-artery stents. N Engl J Med 2006;354:483-95

7. Ramcharitar S, Gaster AL, Daemen J, et al. Drug-eluting stents, restenosis and revascularization. Herz 2007;32: 287-95.

8. Bertrand ME, Rupprecht HJ, Urban P, et al. Double-blind study of the safety of clopidogrel with and without a loading dose in combination with aspirin compared with ticlopidine in combination with aspirin after coronary stenting: the Clopidogrel Aspirin Stent International Cooperative Study (CLASSICS). Circulation 2000;102:624-9.

9. Wenaweser P, Rey C, Eberli FR, et al. Stent thrombosis following bare-metal stent implantation: success of emergency percutaneous coronary intervention and predictors of adverse outcome. Eur Heart J 2005;26:1180-7.

10. Joner M, Finn AV, Farb A, et al. Pathology of drug-eluting stents in humans: delayed healing and late thrombotic risk. J Am Coll Cardiol 2006;48:193-202.

11. McFadden EP, Stabile E, Regar E, et al. Late thrombosis in drug-eluting coronary stents after discontinuation of antiplatelet therapy. Lancet 2004;364:1519-21.

12. Camenzind E, Steg PG, Wijns W. Stent thrombosis late after implantation of first-generation drug-eluting stents: a cause for concern. Circulation 2007;115:1440-55.

13. Moreno R, Fernandez C, Hernandez R, et al. Drug-eluting stent thrombosis: results from a pooled analysis including 10 randomized studies. J Am Coll Cardiol 2005;45:954-9.

14. Bavry AA, Kumbhani DJ, Helton TJ, et al. What is the risk of stent thrombosis associated with the use of paclitax el-eluting stents for percutaneous coronary intervention? A meta-analysis. J Am Coll Cardiol 2005;45:941-6.

15. Bavry AA, Kumbhani DJ, Helton TJ, et al. Risk of thrombosis with the use of sirolimus-eluting stents for percutaneous coronary intervention (from registry and clinical trial data). Am J Cardiol 2005;95:1469-72.
16. lakovou I, Schmidt T, Bonizzoni E, et al. Incidence, predictors, and outcome of thrombosis after successful implantation of drug-eluting stents. JAMA 2005;293:2126-30.

17. Ong AT, McFadden EP, Regar E, et al. Late angiographic stent thrombosis (LAST) events with drug-eluting stents. J Am Coll Cardiol 2005;45:2088-92.

18. Kaiser C, Pfisterer M. Sind Stentthrombosen gehäuft be DES? Herz 2007;32:296-300.

19. Steffel J, Tanner F. Biological effects of drug-eluting stents in the coronary circulation. Herz 2007;32:268-73.

20. Takano M, Mizuno K. Angioscopic findings after drug-eluting stent implantation. Herz 2007;32:281-6.

21. Nakazawa G, Finn AV, Virmani R. Vascular pathology of drug-eluting stents. Herz 2007;32:274-80.

22. Eberli FR, Roffi M. Nachbehandlung nach medikamentös beschichteten Stents. Herz 2007;32:301-6.

23. Duckers $\mathrm{HJ}$, Silber $\mathrm{S}$, de Winter $\mathrm{H}$, et al. Circulating endothelial progenitor cells predict angiographic and intravascular ultrasound outcome following percutaneous coronary interventions in the HEALING-II trial: evaluation of an endothelial progenitor cell capturing stent. Eurolnterv 2007; 3:67-75.

24. Erbel R, Böse D, Haude M, et al. Absorbierbare Stents. Eine vielversprechende Neuerung? Herz 2007;32:308-19.

25. Mehta SR, Yusuf S, Peters RJ, et al., Effects of pretreatment with clopidogrel and aspirin followed by long-term therapy in patients undergoing percutaneous coronary intervention: the PCI-CURE study. Lancet 2001;358:527-33.

26. Bhatt DL, Fox KA, Hacke W., et al., for the CHARISMA Investigators. Clopidogrel and aspirin versus aspirin alone for the prevention of atherothrombotic events. N Eng J Med 2006;354:1706-17.

27. Daemen J, Wenaweser P, Tsuchida K, et al. Early and late coronary stent thrombosis of sirolimus-eluting and paclitaxel-eluting stents in routine clinical practice: data from a large two-institutional cohort study. Lancet 2007;369: 667-78.

28. Chan FK, Ching JY, Hung LC, et al. Clopidogrel versus aspirin and esomeprazole to prevent recurrent ulcer bleeding. $\mathrm{N}$ Engl J Med 2005;352:238-44.

\section{Korrespondenz- anschrift}

Prof. Dr. Thomas F.

Lüscher

Direktor, Klinik für

Kardiologie HerzKreislaufZentrum

UniversitätsSpital Zürich

Rämistraße 100

8091 Zürich

Schweiz

Telefon (+41/44) 255-2121,

Fax -4251

E-Mail:karlue@usz.

unizh.ch 